\title{
A Case of Thunderclap Headache during Pregnancy
}

Caroline Peyronnard MD, Elizabeth Leroux MD, Mehdi Gaha MD

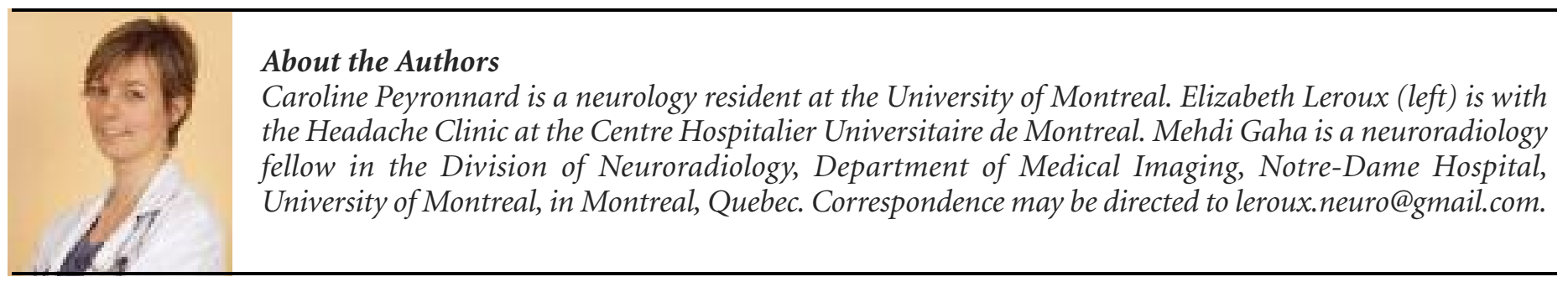

\section{Summary}

Headache during pregnancy is a common issue for which there is a broad differential diagnosis, ranging from common benign primary headaches to certain life-threatening conditions. Although it can be a challenging diagnosis, the presence of previously unknown or new-onset headaches can alert the clinician to the possibility of an underlying secondary process, and orient the subsequent investigation.

Here, we present the case of a 33-year-old migrainous patient presenting in her 36th week of pregnancy with recurrent thunderclap headaches (TCH) in association with multifocal cerebral arterial vasospasms, compatible with a diagnosis of reversible cerebral vasoconstriction syndrome. This case serves as a starting point to briefly discuss this syndrome, its particularities during pregnancy, and its management before reviewing some other important entities that can present with TCH during pregnancy. This case also underscores the paramount importance of identifying the presence of a different pattern of headaches in a patient consulting for this symptom, in order to plan further investigation.

\section{Résumé}

La céphalée durant la grossesse est fréquente et établir le diagnostic peut s'avérer une tâche difficile. Le diagnostic différentiel s'étend de la céphalée primaire bénigne à la céphalée secondaire à un processus mettant en danger la vie du patient. La présence de céphalées nouvelles ou d'apparition récente peut être le signe d'un processus physiopathologique sousjacent, et justifie une investigation subséquente.

L'article présente le cas d'une femme migraineuse de 33 ans à sa 36e semaine de grossesse Présentant des céphalées en coup de tonnerre accompagnées de vasospasmes artériels cérébraux multifocaux, compatibles avec un syndrome de vasoconstriction cérébrale réversible. L'exposé de cas est suivi d'une brève discussion sur ce syndrome, ses particularités durant la grossesse et sa prise en charge, puis de la présentation du diagnostic différentiel des céphalées en coup de tonnerre durant la grossesse. Ce cas illustre l'importance primordiale de cerner les caractéristiques cliniques particulières des céphalées du patient afin d'orienter la démarche diagnostique. 


\section{Case}

A 33-year-old primiparous woman presented to the outpatient gynecology clinic complaining of severe headaches. She was 36 weeks' pregnant, and her medical history consisted mainly of migraines with and without visual aura since the age of 10 years. She had, in the past, tried several triptans, with minimal efficacy, and had noted a recrudescence of her migraines since becoming pregnant, for which she occasionally took acetaminophen. She had an otherwise-normal pregnancy and did not use any other medication. There was no history of illicit drug use.

She complained of recurring episodes of sudden severe throbbing headaches, with a maximal intensity reached in less than 10 seconds. The pain occasionally radiated posteriorly in the neck and lasted a few hours, and the character of the headaches, as well as their explosive onset, differed markedly from her usual migraines. Her general and neurological examinations when first seen at the clinic were unremarkable, apart from a high blood pressure (BP 152/88 $\mathrm{mm} \mathrm{Hg}$ ) that was deemed secondary to her pain. After a pre-eclampsia workup that proved negative, she was discharged with a prescription of metoclopramide and was told to monitor closely her blood pressure.

She came back again 10 days later complaining of continuing severe, recurrent headaches unalleviated by metoclopramide and acetaminophen, and she was admitted for assessment by the neurology team. A pre-eclampsia workup was ordered again and was unremarkable, with the exception of an elevated urinary protein-to-creatinine ratio. In the light of the patient's recurrent thunderclap headaches (TCHs), a diagnosis of reversible cerebral vasoconstriction syndrome (RCVS) was strongly suspected. After normal results were seen on an emergency cerebral computerized tomography, the patient underwent a cerebral magnetic resonance with arteries and veins angiography (MRI-MRA) that confirmed the presence of multifocal narrowings of the vessels in the anterior and posterior circulations compatible with RCVS, without any accompanying brain parenchyma abnormalities. No posterior reversible encephalopathy syndrome (PRES) was seen (Figure 1).

A control showed an elevated protein-to-creatinine ratio and high BP. A diagnosis of severe pre-eclampsia was made. The patient was started on oral nimodipine for the RCVS and intravenous (IV) $\mathrm{MgSO}_{4}$ for the pre-eclampsia. Labour was induced quickly thereafter. After receiving an early epidural anesthesia, our patient gave birth to a healthy baby without complications.

The patient then experienced rare headaches that were not thunderclap in the postpartum period and underwent a control brain MRI-MRA on day 7 that showed complete resolution of the multifocal narrowings and a very small $(6 \times 7 \mathrm{~mm})$ hemorrhagic pituitary adenoma, retrospectively present on the initial imaging. Pituitary lesions are associated with TCHs but not recurrent, and should not cause multifocal vasospasm. The contribution of this finding to the clinical picture is therefore difficult to determine precisely. She was continued on oral nimodipine for another
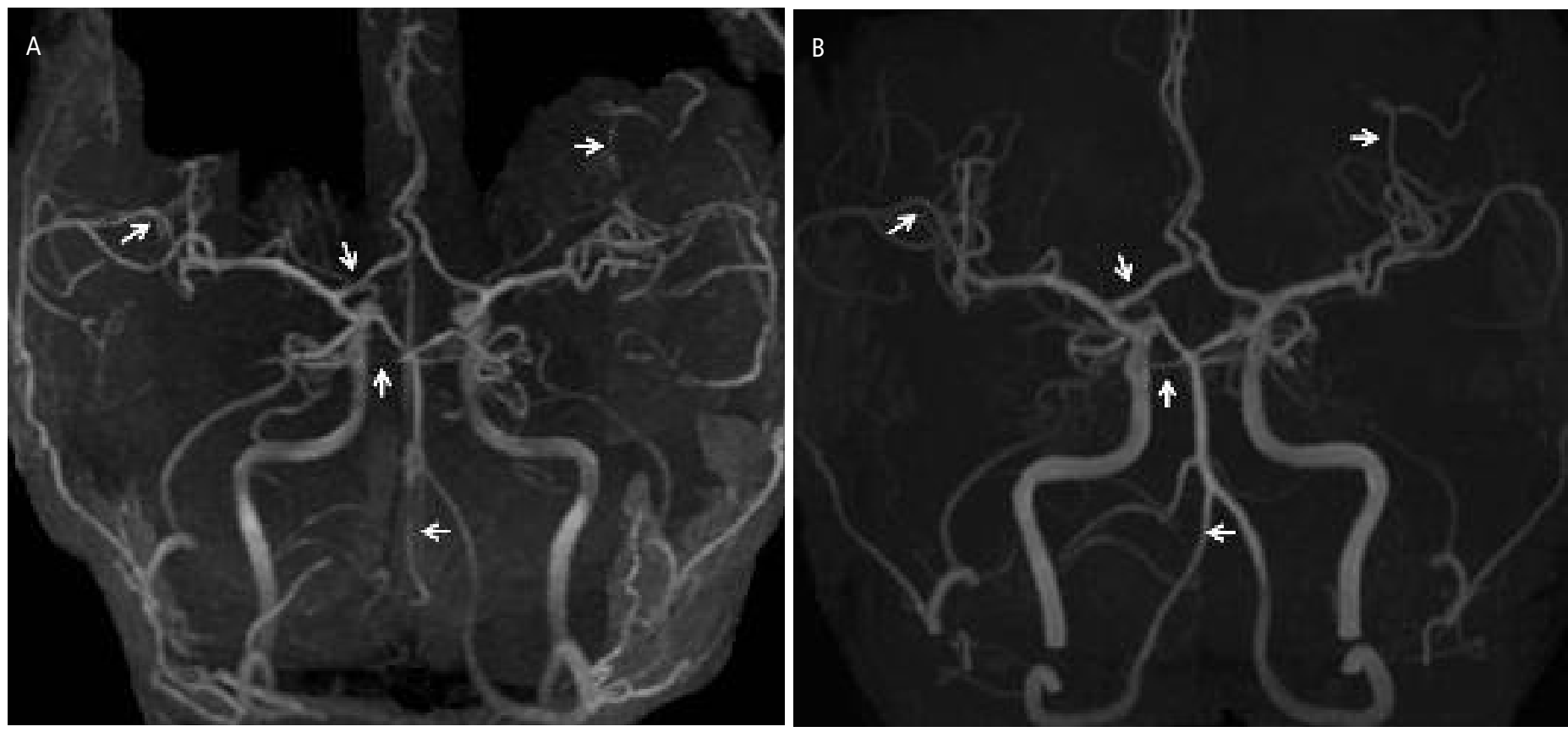

Figure 1. A, Magnetic resonance angiography (MRA) initially showed diffused narrowing of intracranial arteries (arrows) including branches of middle cerebral arteries, the pre-communicant segment of right cerebral anterior artery, the right superior cerebellar artery, and the intracranial right vertebral artery. $B$, An MRA during the follow-up 7 days later demonstrated the resolution of cerebral arterial vasoconstriction (arrows). The patient was taking nimodipine. 
5 weeks and was able to discontinue the treatment without any recurrence of her symptoms. The patient was oriented to our high-risk pregnancy team for future pregnancies.

\section{Discussion}

Headache during pregnancy can represent a diagnostic challenge, encompassing a spectrum of diseases from the more benign primary headache disorders to certain life-threatening conditions. Although a complete review of the many causes of headaches during pregnancy is beyond the scope of this article, we suggest a clinical approach to headaches in the pregnant woman, with a focus on TCHs.

An important clinical clue for the presence of a benign primary headache is a history of previous similar headaches, whereas an unusual or recent-onset headache suggests a more worrisome underlying disorder. The first question to ask a patient presenting for an acute headache should then be, "Is this new or different from your previous headaches?" In the present case, the identification of a new pattern of headaches might have lead to an earlier diagnosis. Any new headache should be considered for an investigation, whereas headaches known to the patients are more likely to be migraine or tension-type headache, the most common primary headaches.

\section{Migraine}

Migraine is a common primary headache disorder affecting predominantly women of childbearing years. ${ }^{1}$ During pregnancy, the stabilization of estrogen levels, as well as the possible contribution of elevated endorphins, ${ }^{2,3}$ leads to a significant improvement in approximately $60-70 \%$ of migrainous patients, ${ }^{4}$ especially in those suffering from migraine without aura. ${ }^{1,4}$ Patients suffering from migraine with aura seem less likely to improve and, since cortical spreading depression is enhanced by high estrogen levels, ${ }^{5}$ it is not rare for migraine with aura to present for the first time during pregnancy. ${ }^{6}$ The rapid fall in estrogen that occurs during the postpartum is conversely associated with a recurrence of headaches in about $34 \%$ of patients in the 1 st week, and 55\% in the 1 st month. ${ }^{1}$

\section{Thunderclap Headaches and RCVS}

In case of a new headache, the clinician should then determine if it is "thunderclap" or progressive. The history should be obtained with open questions first such as, "How did the headache appear?" or, "What were you doing when it appeared, and how did you react?" The question, "Is this the worst headache you ever had?" is not specific enough. $\mathrm{TCH}$, as was the case in our patient, is defined by the International Headache Society ${ }^{7,8}$ as severe headaches reaching a maximal intensity in less than 1 minute, and are the main focus of our discussion. They commonly herald the presence of a serious underlying disorder for which investigation is warranted. Although there is a vast differential diagnosis, ${ }^{8}$ there are certain entities that need to be considered more specifically in the pregnant woman.

\section{Subarachnoid Hemorrhage}

Subarachnoid hemorrhage (SAH) is the first diagnosis to be evoked with a $\mathrm{TCH}$ presentation. Its prevalence is increased in the peri-partum period, ${ }^{9}$ but Kim et al. published that the risk of aneurysmal rupture does not seem to be increased during pregnancy. ${ }^{10} \mathrm{SAH}$ is aneurysmal in $85 \%$ of cases, but other etiologies have to be kept in mind. ${ }^{11}$ The presence of a cortical, non-aneurysmal subarachnoid hemorrhage does not discard the diagnosis of RCVS.

\section{Reversible Cerebral Vasoconstriction Syndrome}

RCVS is an increasingly recognized syndrome, previously called PPA (postpartum angiopathy) when occurring in the postpartum. It is characterized by recurrent TCHs in association with multifocal reversible segmental vasoconstriction of the cerebral arteries. This finding can, however, be missed on initial imaging if performed in the first 7-10 days after the inaugural $\mathrm{TCH}$, the vasospasm being possibly confined to the very small arteries in this early stage. If clinically suspected, imaging should be repeated 7-10 days later. Multiple TCHs are unlikely to be caused by aneurysmal subarachnoid hemorrhage and should strongly suggest the diagnosis of RCVS. The putative underlying mechanisms of RCVS include a transient failure of cerebrovascular tone as well as endothelial dysfunction, ${ }^{12,13}$ which seem to be shared by other pregnancy-associated diseases, such as eclampsia/PRES. Recent data suggest a significant clinical and radiological overlap between PPA and eclampsia/PRES ${ }^{14,15}$ that draws some authors to suggest that they are possibly different manifestations of the same underlying process. ${ }^{14}$ Around 9\% of RCVS cases are related to the postpartum period. ${ }^{16}$ To our knowledge, our patient is the first case to be published with an ante-partum presentation of RCVS. Although RCVS is considered a rather benign entity, seizures, strokes, as well as cortical subarachnoid hemorrhage complicate the clinical course in a significant proportion of patients. ${ }^{15}$ According to two small series of postpartum-related RCVS, the most frequent complications are focal neurological deficits (50-60\%), seizures (28-54\%), intracranial hemorrhage (39\%), encephalopathy (33-60\%), and ischemic strokes $(27-29 \%))^{10,11}$

\section{Cerebral Venous Thrombosis}

Cerebral venous thrombosis needs to be considered in the 
differential diagnosis of TCH, especially during the 3rd trimester of pregnancy and the postpartum, with their accompanying hypercoagulable state. A search for an underlying thrombophilia unrelated to pregnancy is nonetheless warranted in these patients. ${ }^{1}$ Although it can present solely with headaches, it is usually accompanied by other features such as vomiting, a change in consciousness, papilledema, or focal deficits. Magnetic resonance venography remains the gold standard ${ }^{17}$ for diagnosis, and treatment consists of anticoagulation with low molecular weight heparin throughout pregnancy.

\section{Pituitary Apoplexy}

Pituitary apoplexy seems to be a rare but serious entity during pregnancy that presents with $\mathrm{TCH}$ accompanied by visual changes and ophthalmoplegia, usually in the context of an underlying pituitary adenoma. ${ }^{13}$ Estrogens have been shown to induce hyperemia of the hypophysis in an experimental model and could play a role in the underlying pathophysiology. ${ }^{13}$ Replacement of deficient hormones is essential, but the need for rapid surgery remains a matter of debate. ${ }^{1}$

\section{Hemorrhagic Stroke}

Hemorrhagic stroke, although rare in young women, is almost as frequent as ischemic stroke in pregnancy. ${ }^{1}$ Possible etiologies include pregnancy-induced hypertensive disorders, aneurysmal subarachnoid hemorrhage, arteriovenous malformations, metastasis from an underlying choriocarcinoma, and the abovementioned cerebral venous thrombosis and RCVS. ${ }^{1,9}$ Treatment is adjusted according to the underlying disorder.

\section{Management of RCVS}

The first step in RCVS management is the elimination of any precipitating factor, while instructing the patient to avoid any headache triggers. Although no guidelines exist concerning the management of blood pressure, the general agreement is to avoid hypotension that might be more deleterious in the context of cerebral vasospasm..$^{18}$ No randomized trials have been published on RCVS, and management is based on clinical experience and expert opinion. Derived from evidence published for SAH-related vasospasm, ${ }^{19}$ nimodipine is frequently used in such patients; but although it probably reduces the frequency of headaches, studies suggest it might not alter the duration of the vasoconstriction. ${ }^{16}$ Nimodipine is available in the oral form, but its cost in Canada is high. Oral verapamil can be considered as an alternative. IV nimodipine and magnesium sulphate are options. Intra-arterial nimodipine, milrinone, and verapamil have been reported only in severe cases. Steroid use is not encouraged for RCVS as a series of cases have suggested deterioration associated with its use. ${ }^{20}$

\section{Conclusion}

In conclusion, headache during pregnancy is a relatively common issue for which there are numerous etiologies. The presence of an unusual headache should prompt the clinician to suspect an underlying secondary cause, especially in the context of $\mathrm{TCH}$, that mandates a timely investigation. The rapid identification of an underlying disorder can potentially prevent significant morbidity and mortality.

\section{References}

1. Dixit A, Bhardwaj M, Sharma B. Headache in pregnancy: a nuisance or a new sense? Obstet Gynecol Int 2012;2012:697697.

2. Torelli P, Allais G, Manzoni GC. Clinical review of headache in pregnancy. Neurol Sci 2010;31 Suppl 1:S55-8.

3. Sharma R, Panda A. Ondansetron-induced headache in a parturient mimicking postdural puncture headache. Can J Anaesth 2010;57:187-8.

4. Macgregor EA. Headache in pregnancy. Neurol Clin 2012;30:835-66.

5. Eikermann-Haerter K, Kudo C, Moskowitz MA. Cortical spreading depression and estrogen. Headache 2007;47 Suppl 2:S79-85.

6. Cupini LM, Matteis M, Troisi E, et al. Sex-hormone-related events in migrainous females. A clinical comparative study between migraine with aura and migraine without aura. Cephalalgia 1995;15:140-4.

7. The International Classification of Headache Disorders: 2nd edition. Cephalalgia 2004;24 Suppl 1:9-160.

8. Schwedt TJ, Matharu MS, Dodick DW. Thunderclap headache. Lancet Neurol 2006;5:621-31.

9. Bateman BT, Olbrecht VA, Berman MF, et al. Peripartum subarachnoid hemorrhage: nationwide data and institutional experience. Anesthesiology 2012;116:324-33.

10. Kim YW, Neal D, Hoh BL. Cerebral aneurysms in pregnancy and delivery: pregnancy and delivery do not increase the risk of aneurysm rupture. Neurosurgery 2013;72:143-9; discussion 50.

11. van Gijn J, Rinkel GJ. Subarachnoid haemorrhage: diagnosis, causes and management. Brain 2001;124:249-78.

12. Chik Y, Hoesch RE, Lazaridis C, et al. A case of postpartum cerebral angiopathy with subarachnoid hemorrhage. Nat Rev Neurol 2009;5:512-6.

13. Ducros A. Reversible cerebral vasoconstriction syndrome. Lancet Neurol 2012;11:906-17.

14. Fletcher JJ, Kramer AH, Bleck TP, Solenski NJ. Overlapping features of eclampsia and postpartum angiopathy. Neurocrit Care 2009;11:199-209.

15. Fugate JE, Ameriso SF, Ortiz G, et al. Variable presentations of postpartum angiopathy. Stroke 2012;43:670-6.

16. Singhal AB, Hajj-Ali RA, Topcuoglu MA, et al. Reversible cerebral vasoconstriction syndromes: analysis of 139 cases. Arch Neurol 2011;68:1005-12.

17. Demir CF, Inci MF, Ozkan F, et al. Clinical and radiological management and outcome of pregnancies complicated by cerebral venous thrombosis: a review of 19 cases. J Stroke Cerebrovasc Dis. Epub 2012 Aug 15; http://www.sciencedirect.com/science/article/pii/S1052305712002145.

18. Chen SP, Fuh JL, Wang SJ. Reversible cerebral vasoconstriction syndrome: current and future perspectives. Expert Rev Neurother 2011;11:1265-76.

19. Gilsbach JM, Reulen HJ, Ljunggren B, et al. Early aneurysm surgery and preventive therapy with intravenously administered nimodipine: a multicenter, double-blind, dose-comparison study. Neurosurgery 1990;26:458-64.

20. Yancy H, Lee-Iannotti JK, Schwedt TJ, Dodick DW. Reversible cerebral vasoconstriction syndrome. Headache 2013;53:570-6. 